\title{
RNAi Technology and Investigation on Possible Vaccines to Combat SARS-CoV-2 Infection
}

\author{
Pratik Talukder ${ }^{1}$ (D) Sounak Chanda ${ }^{1}$ \\ Received: 7 November 2020 / Accepted: 22 March 2021/ \\ Published online: 7 April 2021 \\ (C) The Author(s), under exclusive licence to Springer Science+Business Media, LLC, part of Springer Nature 2021
}

\begin{abstract}
Coronavirus disease of 2019 (COVID-19) pandemic, taking place globally, occurs as a result of the SARS-CoV-2 viral infection which has caused death of innumerable numbers of people and is responsible for a massive drop in the global economy. Millions of people are infected, and the death rate is also quite high in different countries. So, there is an urgent requirement of the invention of some effective and efficient drugs that can be effective against this deadly viral infection. The invention of new drugs and vaccine has become a matter of utmost importance to stop the mayhem of coronavirus pandemic. In the middle of such a deadly pandemic, the necessity of development of a vaccine is of high importance in this context. Among all the popular methods of vaccine development, the mRNA vaccines turned out to be the one of the most versatile vaccine with quick responses. However, in this review, we have explained all the possible types of vaccines available including DNA vaccines, RNA vaccines, and live and attenuated vaccines. Their effectiveness, importance, and application of the vaccines against the SARS-CoV-2 virus have been discussed. Research is also being conducted in the field of gene silencing, and one of the best possible ways to combat the virus at the molecular level is by applying RNAi technology. The modified siRNA molecules can be used to silence the gene expression of the virus. A summarization of the virus's behavior, characteristics, and the methods by which RNAi technology can be administered to control the virus is depicted in this study.
\end{abstract}

Keywords COVID-19 $\cdot$ Vaccine $\cdot$ ACE2 receptors $\cdot$ Antibody $\cdot$ Antigen $\cdot$ siRNA $\cdot$ Gene silencing

\section{Introduction}

One of the greatest pandemic in human civilization is caused by the SARS-CoV-2 virus or the novel coronavirus infection, and it is still causing havoc throughout the world [1].

Pratik Talukder

pratiktalukder@gmail.com

1 Department of Biotechnology, University of Engineering and Management, University Area, Plot, Street Number 03, Action Area III, B/5, Newtown, Kolkata, West Bengal 700156, India 
Pharmaceutical industries, government agencies, scientists, and researchers from all over the world are tirelessly working for creating a new vaccine effective against this deadly viral infection. The whole world is suffering from this pandemic and trying to find a way out; all the pharmaceutical companies are trying their best to find a cure of the pandemic by inventing a vaccine. SARS-CoV-2, an envelope, positive-sense, single-stranded RNA beta coronavirus, is a member of the family corona viridae [2]. The RNA is associated with a nucleoprotein, and it is encapsulated within a helically symmetrical nucleocapsid made up of matrix protein. Recent cryo-electron microscopy tomography studies reported that this virus is $125 \mathrm{~nm}$ in diameter [3]. The most unique and defining feature of this virus is the presence of the club-shaped spike projections. These spike proteins give the virus the appearance of solar corona, and thus was named so. Coronavirus belongs to the order nidovirales, a group of large, non-segmented, positive-sense, and single-stranded RNA animal viruses [4]. During the later half of 2019, many patients with severe pneumonia were admitted to Wuhan City Hospital in China [5]. The pneumonia causing viral agent was sequenced, and the results came out to be a strain of beta coronavirus which was almost a similar strain like SARS-like BAT coronavirus, bat-SL-COVZC45 and bat-SLCOVZXC21 with $88 \%$ similarity, $79.5 \%$ homology with SARS-CoV-1, and 50\% with MERS-CoV [6]. Although the mortality rate is estimated to be lower than that of SARSCoV-1 or MERS-CoV, coronaviruses can have negative impact on different organs of human body and cause a large variety of diseases in mammals, including livestock, domesticated animals like dogs and cats, as well as primates other than human. Human coronaviruses are commonly associated with mild respiratory and enteric diseases, although they are also known to cause more critical lower respiratory tract illnesses [7]. Studies on sequence similarity among different viruses showed a 79\% similarity of SARS-CoV-2 with SARS-CoV-1 and about 50\% with MERS-CoV [3]. MERS is a viral respiratory disease which occurs because of Middle East Respiratory Syndrome Coronavirus or MERS-CoV [8]. This virus was first identified in Saudi Arabia in 2012. According to the World Health Organization, approximately $35 \%$ of patients having infection with MERS-CoV have died [9]. The MERS-CoV infection generally occurs from human to human contact, especially in health care sectors, but some studies also suggest that dromedary camels can be a vital host or carrier of the MERS-CoV and so interactions with camels can also cause this disease [10]. However, the exact mode of transmission process of MERS-CoV virus from dromedary camels to humans is still unknown. This virus does not pass from a person to another person unless they are in very close contact. The largest outbreak of this disease is seen in countries like Saudi Arabia, United Arab Emirates, and the Republic of Korea. MERS-CoV is a zoometric virus. This indicates that this virus is generally transmitted between animals and people. This virus is also identified in many dromedaries from many countries of South and West Asia and Northern Africa [11]. Apart from developing SARS-CoV-2 vaccine, the focus is also given on finalizing the appropriate dose of it [12]. Both pharmacokinetics and pharmacodynamics studies are carried out to determine the optimum dose [13]. In coronavirus disease of 2019 (COVID-19) vaccine trials, it is observed that the vaccines are capable of inducing immune response and producing antibodies that will bind and neutralize the SARS-COV-2 virus [14]. Research is going on to optimize the vaccine which will induce the production of ample amount of antibody which will render long-term protection [15]. Apart from protein-based vaccines, research to develop mRNA vaccine is also underway, and if it succeeds, it will revolutionize the whole outlook of vaccine research as very few RNA-based vaccines are successful. 


\section{Structure and Genomic Organization of the Virus}

The SARS-CoV-2 genomic RNA is non-segmented, positive sense and is about 29,700 bp in length and has several large open reading frames (ORFs) [16]. The genome contains a $5^{\prime}$ cap structure along with a $3^{\prime}$ poly (A) tail, allowing it to act as an mRNA for translation of the replicase polyprotein. Out of the total genome size, around $20 \mathrm{~kb}$ code for the non-structural proteins (NSPS), while the rest are structural or accessory genes. The SARS-CoV-2 genomic RNA is encapsulated by multiple nucleocapsid proteins [17]. It has the following domains: 5'leader-UTR- replicase-S (Spike)-E (Envelope)-M (Membrane) - N (Nucleocapsid)-3' UTRpoly (A) tail with accessory genes combined within the structural genes at the $3^{\prime}$ end of the genome. The most abundant structural protein in the virion is the membrane (or the $\mathrm{M}$ ) protein. The protein is small in size (about 25 to $30 \mathrm{kDa}$ ) with three transmembrane domains and gives the virion its shape [18]. It has a small $\mathrm{N}$-terminal glycosylated ectodomain and a much larger C-terminal endodomain that extends 6-8 nm into the viral particle. The spike glycoprotein is about of $140 \mathrm{kDa}$, membrane glycoprotein is of around $23 \mathrm{kDa}$, and the envelope protein is approximately of $10 \mathrm{kDa}$ [19]. The arrangements of nucleocapsid protein (N), envelope protein (E), and membrane protein (M) among other beta coronaviruses are different [20]. The envelope has club-shaped glycoprotein projections. Besides these main structural proteins, different COVs encode special structural and accessory proteins, such as a hemagglutininesterase protein $3 \mathrm{a} / \mathrm{b}$ protein and $4 \mathrm{a} / \mathrm{b}$ protein [21]. Most of the accessory proteins are not associated with replication process; however, they have important functions in viral pathogenesis [22]. The most abundant structural protein is the membrane (M) glycoprotein which spans the cell membrane bi-layer three times, leaving a short $\mathrm{NH}_{2}$-terminal domain outside the virus and a long $\mathrm{COOH}$ terminal cytoplasmic domain inside the virus [23]. Molecular structure of the virus is shown in Fig. 1.

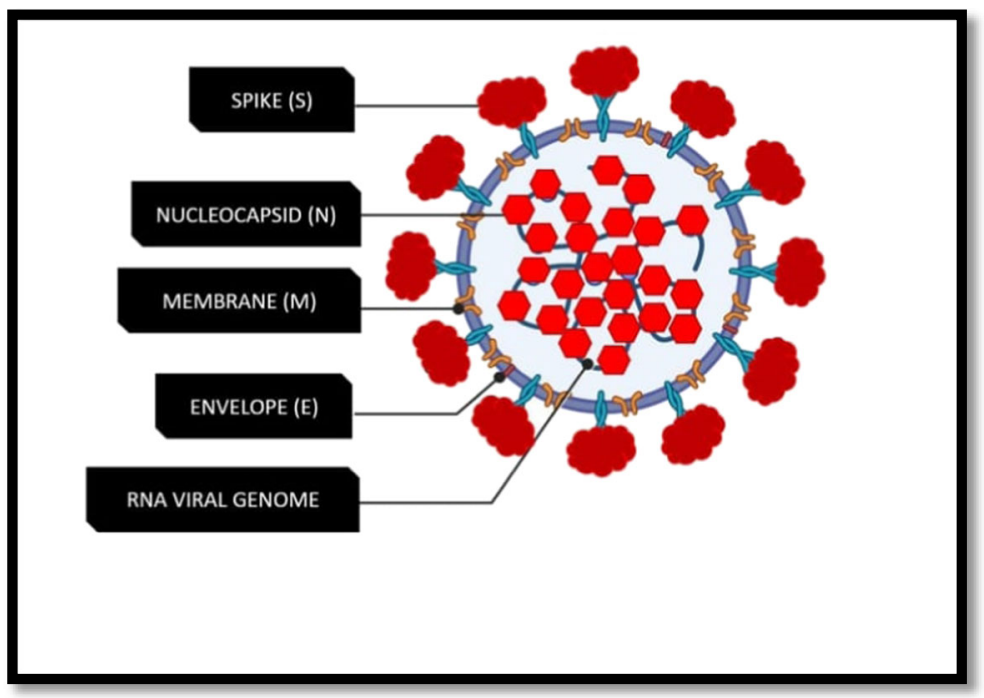

Fig. 1 Molecular architecture of SARS-CoV-2 with both outer surface and inner core structures [24] 


\section{Transmission of SARS-CoV-2}

When the spread of SARS-CoV-2 began to start, it was confined within China only. The USA has the highest number of confirmed cases until now; India and China being the most populated countries are trying to restrict the spreading through complete lockdown process in their respective countries [25]. Similarly, the UK has also tried to maintain a low rate graph by maintaining social distancing and lockdown. The studies and research show that the presence of multiple strains and high mutation capability of the virus made it more difficult to contain the spread. The disease is spread by the droplet transmission mainly [26]. The infected people should follow strict quarantine for a definite span of time [27].

\section{Prospect of RNAi-Mediated Technology for Targeting SARS-CoV-2}

Since its discovery in in the last decade of the twentieth century, RNAi technology has become a handy tool for suppressing the target genes responsible for viral pathogenesis, viral progression, and virulence. Specific binding of siRNA and shRNA can silence the target molecules. Several RNAibased drugs can be repurposed to treat SARS-CoV-2 infection. Several studies reported that siRNA molecules have the potential to target SARS-CoV-2 and MERS-CoV viruses. The first successful siRNA-mediated inhibition of SARS-CoV-1 was reported by Weimin et al. (2003). To combat SARS-Cov-2 infection, RNAi approach can be used to either target the viral proteins necessary for survival and replication of SARS-CoV-2 or the receptor proteins of the host cells which interact with the viral envelop. Hence, RNAi-based approach can either be host target-specific or viral targetspecific. Angiotensin-converting enzyme 2 (ACE 2) receptors of the host cell are used to internalize the SARS-CoV-2 virus [28]. Hence, the ACE 2 receptor can be a potential target. If these receptors can be blocked, the entry and further progression of the virus can be restricted. Deletion mutation in $A C E 2$ prevents the formation of the receptors and thus inhibits the virus from binding the ACE2 receptors and prevents its entry and further infection. Proteolytic cleavage of the external domain of ACE 2 can render it functionless; but as ACE 2 has a beneficial role in our body targeting it and silencing it, it also has a negative impact in humans. Under these circumstances, it is more prudent to target viral proteins rather than the host proteins. All the major types of SARS-CoV-2 surface proteins - S, E, M, and $\mathrm{N}$ - can be potential targets of RNAi-induced silencing [7]. Both the endo and exodomains of $\mathrm{M}$ proteins of the virus can be targeted for RNAi-mediated silencing approach as these proteins are needed to maintain viral membrane integrity. It was also observed that siRNAmediated targeting was also successful against the gene which encodes the viral M protein of SARS. It is already reported in several studies that the targeting of specific conserved domains of the genes of SARS-CoV-2 that encode virus helicase, RNA-dependent RNA polymerase, proteolytic enzyme, and nucleoprotein can reduce the viral load up to $70 \%, 50 \%, 90 \%$, and $50 \%$ respectively [29]. Several biotechnology companies are trying to create a library of siRNAs to target SARS-COV-2. Two USA-based companies Vir Biotech and Alnylam Pharmaceuticals are working jointly in this field. It is reported that about 350 siRNAs are already synthesized which can target SARS-CoV-2; among these, 30 siRNA designs are already submitted for patent filing [30]. Most of the patented siRNA molecules are targeted against the viral replicase and RNA polymerase [31]. Studies show that the efficiency of these siRNAs in killing or inhibiting the target virus ranges from 50 to $90 \%$ [32]. Apart from that, Olix Pharmaceuticals of South Korea also designed siRNA targeting highly conserved domains of SARS-CoV-2 RNA. The gene which codes 3 chymotrypsin-like protease $(3 \mathrm{CL})$ is highly conserved among the different strains of SARS-CoV-2 and MERS-CoV virus. 
Hence, it can be considered one of the potential target for RNAi-induced gene silencing [29]. siRNA can be delivered by the use of nanocarriers [33]. Among the delivery systems which can be used to deliver siRNA to target the genes of SARS-CoV-2 and MERS-CoV, nanoparticle-based carriers and viral vectors have shown the highest efficiency [34]. In an in vitro study, it was observed that aerosol-based delivery system-coupled polydendrimer nanocarriers can be used for smooth and efficient delivery of siRNA on to the lung epithelial cells to target SARS-CoV-2 [35]. A schematic diagram of RNAi-mediated mechanism for targeting SARS-CoV-2 is depicted in Fig. 2.

\section{Type of Vaccines and Their Mode of Action}

\section{Live or Attenuated Vaccine}

This type of virus is formed of a whole virus which is to be neutralized. Some of the examples are chickenpox vaccine, MMR, etc. [37].

The approach allows specific binding and silencing of the targets by using siRNA and shRNA molecules.

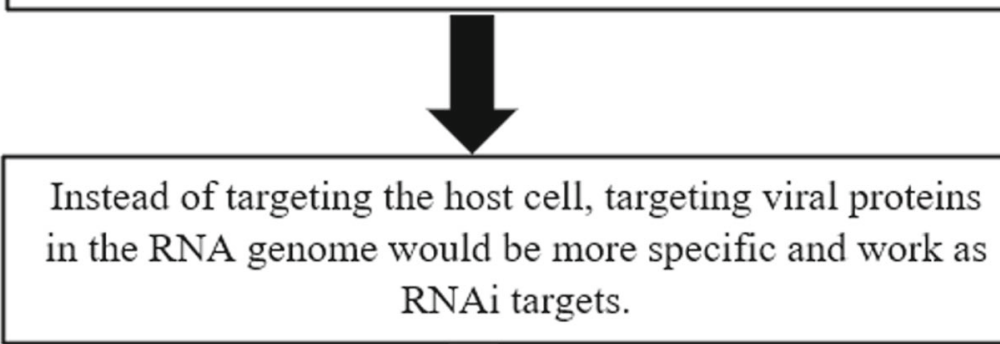

Instead of targeting the host cell, targeting viral proteins RNAi targets.

Delivering siRNA using stable nucleic acid lipid particles can inactivate the virus.

Targeting the RNA-dependent RNA polymerase gene showed effective result in silencing the gene using siRNA.

Fig. 2 Scheme of RNAi-mediated mechanism for targeting SARS-CoV-2 


\section{Inactivated or Killed Vaccine}

These types are made from dead viruses which are not capable of causing the infection alone. Polio vaccine can is an ideal example [38].

\section{Toxoid Vaccine}

These types are mainly made from bacterial toxins, which are inactive in nature. Tetanus can be considered an ideal example [39].

\section{Conjugate or Subunit Vaccine}

These types of vaccines are made from a particular part of the virus which is generally a key protein. This key protein is extracted from a live virus usually or it is otherwise synthesized using recombinant DNA technology. Hepatitis B can be considered an ideal example [40].

\section{DNA Vaccines}

The vaccine industry has increased and developed themselves over the years. New kinds of vaccine types are developed. DNA vaccine has come out specifically as an exception to the traditional vaccines [41]. The mode of action is different; it is done by introducing the genetic sequence of a viral antigen into the host cells. The B lymphocytes of our body can target these genetic materials for the purpose of adaptive immunity [42].

\section{RNA Vaccines}

\section{RNA Vaccines}

RNA vaccines work by introducing an mRNA sequence that can be synthesized in the laboratory using a DNA sequence which codes for a disease-specific antigen. When the synthesized RNA molecule is introduced within the body, it uses the cellular machinery to translate itself in order to produce proteins which resemble the antigen and is expressed in the cellular surface, which can be recognized by the immune system, triggering specific responses and memory. RNA vaccines are faster and cheaper to produce than traditional vaccines, and therefore is a viable candidate for vaccine generation in case of viral diseases which require urgent discovery of vaccine. RNA is unstable compared to DNA, and it has a tendency to degrade rapidly. Due to their shorter half-life and degrading nature, the application of RNA in vaccine technology is limited. During clinical trials of many early RNA vaccines, it was observed that results were ineffective and the productivity was not enough and failed to give rise to a robust immune response [43]. It can be due to the improper integration with the cells of the body. With advancement of technologies in molecular biology, the efficiency of RNA vaccines has enhanced. Advancement of technology enabled the protection of the RNA from early degradation so that there is enough time for the immune system to recognize it as a foreign molecule and develop an immune response against it. The mode of action RNA vaccine is almost similar to that of DNA vaccine, but the efficacy is lesser because it is highly unstable [44]. Among all the popular modes of vaccines, the mRNA vaccines turned out to be one of the most versatile vaccines with quick responses. Apart from protein-based vaccines, 
research to develop mRNA vaccine is also underway, and if it succeeds, it will revolutionize the whole outlook of vaccine research, as very few RNA-based vaccines are successful. An mRNA vaccine is composed of an mRNA strand. This codes for an antigen which is diseasespecific [45]. Moderna, a pioneer in vaccine production, has already reported a positive impact of mRNA vaccines in combating SARS-CoV-2 infection [46]. They also reported that mice which were administered with mRNA encoding the full-length of SARS-CoV-2 had shown higher rate of antibody production compared to mRNA encoding only the $\mathrm{S}$ or $\mathrm{S} 2$ subunit. The same kind of experiment was carried out in New Zealand in white rabbits, and it has also shown the same kind of results [47]. The National Institute of Allergy and Infectious Disease in collaboration with Moderna reported that mRNA-1273, an mRNA-based vaccine, is showing promising results in combating SARS-CoV-2 infection [48]. Different mRNAbased vaccines are designed based on the sequences of the spike protein, envelope protein, membrane protein, and nucleocapsid protein and are effectively working and providing immunogenic response to combat the disease [39]. The mechanism of antibody production by RNA vaccine is depicted in the Fig. 3.

\section{COVID-19 Vaccine Research}

A vast range of approaches including nucleic acid (DNA and RNA), virus-like particle, peptide, recombinant protein, live attenuated virus, and inactivated virus approaches are adopted for generating SARS-CoV-2 vaccines. Vaccines which are dependent on viral vectors offer higher level protein expression and long-term stability and help in inducing strong immune responses. Adjuvants can increase the immune response and can make lower doses viable and thus will help in vaccination [49]. So far, at least 10 developers are in the race for developing adjuvant vaccines against the coronavirus [50].

It is of utmost importance to identify which vaccine and adjuvant can have protective antibody response against SARS-CoV-2 [51]. It has been observed that immunization of mice with inactive SARS-CoV-2, immunization of rhesus macaques with MVA-encoded S protein, and also the immunization of mice with DNA vaccine encoding full-length S protein can induce antibody-

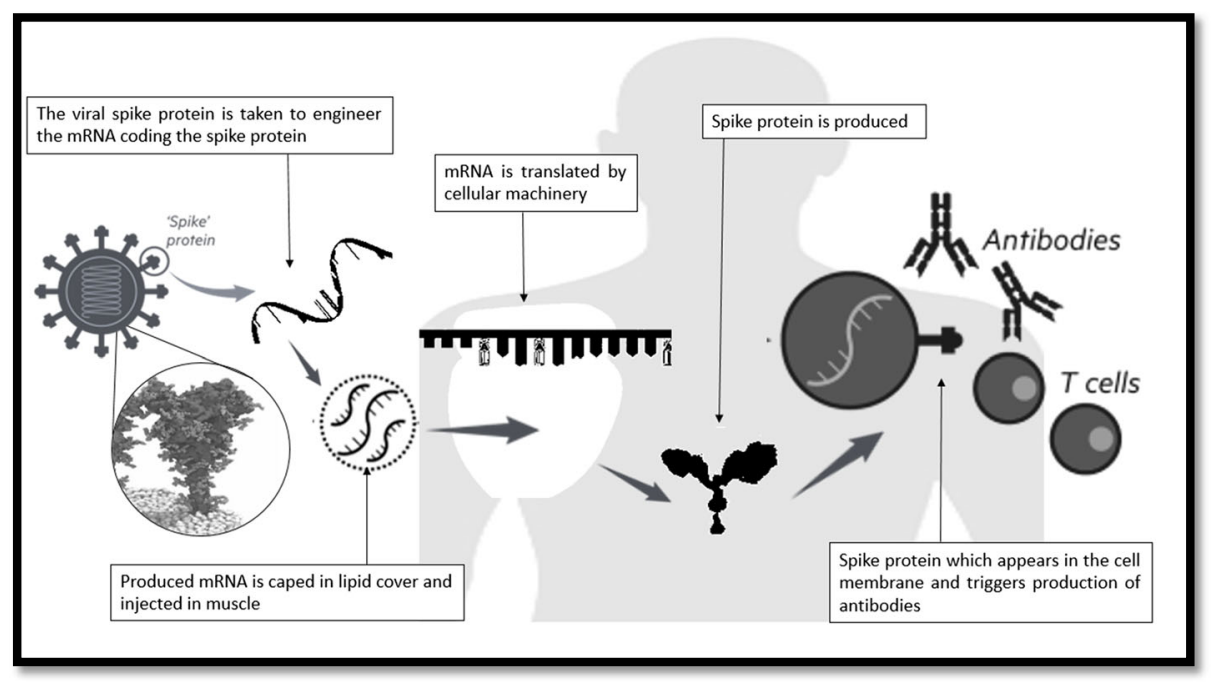

Fig. 3 The mechanism of antibody production by RNA vaccine [36] 
dependent enhancement (ADE) to some extent [52]. It is also reported that double-inactivated SARS-CoV-2 vaccine has been able to neutralize antibody responses in aged mice. On the other hand, studies in mice also showed that a peptide vaccine which focuses against specific epitopes within the RBD of the S protein has protective antibody responses [53]. Also, live attenuated SARS-CoV-2 vaccine can induce protective immune responses in aged mice and hence can further enhance vaccine efficiency [54]. Viral vector vaccines targeted against coronavirus's spike protein and nucleocapsid protein give anti $\mathrm{S}$ and anti $\mathrm{N}$ IgG in immunized mice respectively. Now, there have been multiple vaccine candidates (including nucleic acid vaccines, viral vector vaccines, and subunit vaccines) in the preclinical and clinical trial stages.

Some of the vaccine candidates which have received approvals for phase I and II clinical trials by the World Health Organization are listed below.

\section{Chadox1 Vaccine}

The UK has been working on their one of the major COVID-19 vaccine projects. This currently is under development and under testing by the University of Oxford. When applied in rhesus macaque monkeys, positive results are observed with no adverse effects. The ChAdOx 1 nCOV-19 provokes a $\mathrm{T}$ cell response within 14 days of vaccination. It can also induce antibody response within 28 days. A clinical trial program, which was conducted, predicted that in humans, the strongest immune response was seen in the 10 participants who were given dual doses of the vaccine and then that would indicate that it could be a great way and it might be a good strategy for vaccination. In the UK, the first and second phase trials increased in April and the tests of the Oxford coronavirus vaccine ChAdOx1 nCOV-19. The research work for creating this vaccine was started in January 2020. During the first and second phase of the trials, the vaccine has been tested in more than 1000 healthy adult volunteers between 18 and 55 years in a strategically random trial. Between April 23, 2020, and May 21, 2020, 1077 volunteers received the vaccine ChAdOx1 nCOV-19. No health hazards were reported in those volunteers. The third phase trials in the low-income and middleincome countries including Brazil and South Africa have already begun. AstraZeneca remain committed to fulfilling their commitment for broad and equitable access to the vaccine. The last phase of clinical trials will begin and will prove themselves to be successful. This is approved by the UK, the USA, and by Europe's Inclusive Vaccines Alliance, the Coalition for Epidemic Preparedness, and the Serum Institute of India [35].

\section{Sputnik-V Vaccine}

Russia managed to successfully build the first vaccine in the world against the SARS-CoV-2 novel coronavirus named Sputnik-V. The vaccine is named after the first artificial satellite Sputnik-V. Although no data is available on the safety margin and efficiency of the vaccine, the scientists are hopeful that the final phase of those clinical trials will be completed soon. In the first two phases, the vaccine was tested on a lesser number of people. The scientists at the Moscow-based Gamaleya Research Institute of Epidemiology and Microbiology developed this vaccine. Adenovirus 5 and adenovirus 26 were constructed individually for making the spike proteins of SARS-CoV-2. These proteins are on the surface of the virus, and hence, they are the target for antibodies against the SARS-CoV-2 virus [8]. The University of Oxford in research collaboration with AstraZeneca used a chimpanzee adenovirus to develop the vaccine. Another vaccine which is most recently developed by China-based CanSino Biologics Inc. is based on adenovirus 5. The company Johnson \& Johnson 
uses adenovirus 26 for its vaccine development. All these vaccines are undergoing or already have undergone through the initial safety measurements and checked if they possess any serious side effects. In total, 38 people first got a shot containing the engineered adenovirus 26 components [57]. After 3 weeks, those 38 people were given booster injections of the engineered adenovirus 5 components. However, the observations of these studies have not been released yet. As a general scientific expectation, the candidates in those vaccines that have been applied may develop antibodies to the adenovirus carrying the spike protein.

\section{Ad5-nCOVVaccine}

In China, the epicenter of this viral outbreak is also trying to develop its own indigenous vaccine. CanSino Biologics is trying to prepare one vaccine. The vaccine is known as Ad5nCOV. The World Health Organization has announced that the clinical trials are yet to be conducted for 17 vaccine candidates. Out of those, 7 are being developed in China. Out of these, one of the candidates has been made by the Oxford University and AstraZeneca. This vaccine is in its phase III studies. The researchers in China have predicted that the invention of a new drug can be able to cease COVID-19 infection, more than the vaccine can do [8].

\section{COVAXIN Vaccine}

Bharat Biotech successfully developed India's first anti-COVID vaccine called COVAXIN. It is made in collaboration with the National Institute of Virology. COVAXIN has already got an approval from the Drugs Controller General of India for human clinical trials [32]. The phase I of the clinical trials turned out to be successful for this vaccine. The second and third stages are still to be completed and reported. If these stages turn out to be a success too, COVAXIN will be registered as the country's "first" indigenous COVID-19 vaccine. When this vaccine will be ready for mass vaccination, it will have no potential for infection or replication because the virus is already killed. The preclinical tests on laboratory animals like guinea pigs and mice have been already being done to determine its efficacy before the company approached CDSCO for the permission of approval for starting human trials. Twelve institutes have been approved by ICMR where the clinical trial of COVAXIN was done.

Apart from the abovementioned vaccines, work is going on to develop X-CoV2373 vaccine by NOVAVAX, at Witwatersrand University in Johannesburg, South Africa. They will start with the clinical trial soon. Another vaccine AD26 CoV2-S, a Johnson \& Johnson creation, is also under trial. This trial is in collaboration with the Oxford University and the Jenner Institute. BioNTech in collaboration with Pfizer and Fosun Pharma (Chinese drug-maker) are developing an mRNA vaccine which is to be delivered in two doses. In May, they launched a phase I and II trials on two versions of the vaccines. It was observed that both these versions produced antibodies and T-cells when administered in the human body. One version, BNT162b2, showed relatively limited side effects (fevers and fatigue) and so allowed to carry out phase III trials.

\section{Conclusion}

There is no specific drug for the treatment of SARS-CoV-2 infection. At the onset of the pandemic, there were no vaccines or drugs available, and it necessitated the need to having a 
vaccine. The world has got few SARS-CoV-2 vaccines, and the scientists are trying their level best to discover more. Even after the arrival of the vaccine in the market and after its distribution among the civilians for use, research and surveillance process must be carried on for observing and tracking the efficacy of vaccination in the population. Along with it, actors such as the rate of mutation of the virus, generation of new strains, and the long-term effectiveness of the vaccine against all the strains should be checked and looked upon. For the treatment of SARS-CoV-2 infection, RNAi-based therapy can be developed against the novel coronavirus SARS-CoV-2. RNA interference (RNAi) mechanism can be utilized to formulate an antidote against the harmful virus. It specifically binds and silences targets by using siRNA and shRNA molecules. In this review, we lay out the prospects of RNAi technology for combating SARS-CoV-2 emphasizing on viral delivery approaches and molecular underpinning of design of RNAi agents is summarized. The future of RNAi therapy is of utmost importance as it is incredibly beneficial for combating SARS-CoV-2. This review could help to achieve a better understanding of the various aspects of possible vaccine discovery and use of RNAi technology to combat against SARS-CoV-2 infection.

Acknowledgements The author sincerely acknowledges the University of Engineering and Management.

Code Availability Not applicable

Author Contribution Both the authors Sounak Chanda and Pratik Talukder contributed, and the manuscript was mainly prepared by Pratik Talukder.

Data availability Not applicable

\section{Declarations}

Ethical Approval Not applicable

Consent to Participate Not applicable

Consent for Publication The authors give the consent for publication.

Conflict of Interest The authors declare no competing interests.

\section{References}

1. Chen, Y., Liu, Q., \& Guo, D. (2020). Emerging, and pathogenesis. Journal of Medical Virology, 92(4), $418-423$.

2. Yount, B., Curtis, K. M., Fritz, E. A., Hensley, L. E., Jahrling, P. B., Prentice, E., Denison, M. R., Geisbert, T. W., \& Baric, R. S. (2003). Reverse genetics with a full-length infectious cDNA of severe acute respiratory syndrome coronavirus. PNAS., 100(22), 12995-13000.

3. Jiang, H., Li, Y., Zhang, H. N., Wang, W., Yang, X., Qi, H., Li, H., Men, D., Zhou, J., \& Tao, S. C. (2020). Global profiling of SARS-CoV-2 specific IgG/IgM responses of convalescents using a proteome microarray. Nature Communications, 11(1). https://doi.org/10.1038/s41467-020-17488-8.

4. De Haan et al. (1998). Coronavirus particle assembly: Primary structure requirements of the membrane protein. Journal of Virology, 172(8). 
5. Kim, J. C., Spence, R. A., Currier, P. F., Lu, X., \& Denison, M. R. (1995). Coronavirus protein processing and RNA synthesis is inhibited by the cysteine proteinase inhibitor. Virology., 208(1), 1-8.

6. Kanjanahaluethai, A., Jukneliene, D., \& Baker, S. C. (2003). Identification of the murine coronavirus MP1 cleavage site recognized by papain-like proteinase 2. Journal of Virology, 77(13), 7376-7382.

7. Mousavizadeha, L., Sorayya Ghasemi, S. (2020). Genotype and phenotype of COVID-19: Their roles in pathogenesis. Journal of Microbiology, Immunology and Infection. https://doi.org/10.1016/j.jmii.2020.03. 022.

8. Lu, R., Zhao, X., Li, J., Niu, P., Yang, B., Wu, H., Wang, W., Song, H., Huang, B., Zhu, N., Bi, Y., Ma, X., Zhan, F., Wang, L., Hu, T., Zhou, H., Hu, Z., Zhou, W., Zhao, L., Chen, J., Meng, Y., Wang, J., Lin, Y., Yuan, J., Xie, Z., Ma, J., Liu, W. J., Wang, D., Xu, W., Holmes, E. C., Gao, G. F., Wu, G., Chen, W., Shi, W., \& Tan, W. (2020). Genomic characterisation and epidemiology of 2019 novel coronavirus: Implications for virus origins and receptor binding. Lancet., 395(10224), 565-574.

9. Bárcena, et al. (2009). Cryo-electron tomography of mouse hepatitis virus: Insights into the structure of the corona virion. PNAS., 106(2), 582-587.

10. Neuman, B. W., Adair, B. D., Yoshioka, C., Quispe, J. D., Orca, G., Kuhn, P., Milligan, R. A., Yeager, M., \& Buchmeier, M. J. (2006). Supramolecular architecture of severe acute respiratory syndrome coronavirus revealed by electron cryomicroscopy. Journal of Virology, 80(16), 7918-7928.

11. Armstrong, J., Niemann, H., Smeekens, S., Rottier, P., \& Warren, G. (1984). Sequence and topology of a model intracellular membrane protein, E1 glycoprotein, from a coronavirus. Nature, 308(5961), 751-752.

12. Engin, A. B., Nikitovic, D., Neagu, M., Henrich-Noack, P., Docea, A. O., Shtilman, M. I., Golokhvast, K., \& Tsatsakis, A. M. (2017). Mechanistic understanding of nanoparticles interactions with extracellular matrix: The cell and immune system. Particle and Fibre Toxicology, 14(1), 22.

13. Delfosse, V. C., et al. (2013). In vivo short-term exposure to residual oil fly ash impairs pulmonary innate immune response against environmental mycobacterium infection. Environmental Toxicology, 30(5), 589596.

14. Doceaao, C. D., et al. (2019). Study design for the determination of toxicity from long-term-low-dose exposure to complex mixtures of pesticides, food additives and lifestyle products. Toxicology Letters, 258, 179.

15. Doceaao, C. D., et al. (2018). Six months exposure to a real life mixture of 13 chemicals below individual NOAELs induced non monotonic sex-dependent biochemical and redox status changes in rats. Food and Chemical Toxicology, 115, 470-481.

16. Jaume, M., Yip, M. S., Cheung, C. Y., Leung, H. L., Li, P. H., Kien, F., Dutry, I., Callendret, B., Escriou, N., Altmeyer, R., Nal, B., Daeron, M., Bruzzone, R., \& Peiris, J. S. M. (2011). Anti-severe acute respiratory syndrome coronavirus spike antibodies trigger infection of human immune cells via a $\mathrm{pH}$ and cysteine protease-independent Fc $\gamma \mathrm{R}$ pathway. Journal of Virology, 85(20), 10582-10597.

17. Yasui, F., Kai, C., Kitabatake, M., Inoue, S., Yoneda, M., Yokochi, S., Kase, R., Sekiguchi, S., Morita, K., Hishima, T., Suzuki, H., Karamatsu, K., Yasutomi, Y., Shida, H., Kidokoro, M., Mizuno, K., Matsushima, K., \& Kohara, M. (2008). Prior immunization with severe acute respiratory syndrome (SARS)-associated coronavirus (SARS-CoV) nucleocapsid protein causes severe pneumonia in mice infected with SARS-CoV. Journal of Immunology, 181(9), 6337-6348.

18. Yip, M. S., et al. (2016). Antibody-dependent enhancement of SARS coronavirus infection and its role in the pathogenesis of SARS. Hong Kong Medical Journal, 22, 25-31.

19. Wang, Q., Zhang, L., Kuwahara, K., Li, L., Liu, Z., Li, T., Zhu, H., Liu, J., Xu, Y., Xie, J., Morioka, H., Sakaguchi, N., Qin, C., \& Liu, G. (2016). Immunodominant SARS coronavirus epitopes in humans elicited both enhancing and neutralizing effects on infection in non-human primates. ACS Infectious Diseases, 2(5), 361-376.

20. Tan, W., et al. (2020). Viral kinetics and antibody responses in patients with COVID-19. Preprint at Medrxiv. https://doi.org/10.1101/2020.03.24.200423822020.

21. Pierson, T. C., Fremont, D. H., Kuhn, R. J., \& Diamond, M. S. (2008). Structural insights into the mechanisms of antibody-mediated neutralization of flavivirus infection: Implications for vaccine development. Cell Host \& Microbe, 4(3), 229-238.

22. Yuan, F., et al. (2005). Influence of Fc $\gamma \mathrm{R}$ IIA and MBL polymorphisms on severe acute respiratory syndrome. Tissue Antigens, 66(4), 291-296.

23. Bolles, M., Deming, D., Long, K., Agnihothram, S., Whitmore, A., Ferris, M., Funkhouser, W., Gralinski, L., Totura, A., Heise, M., \& Baric, R. S. (2011). A double-inactivated severe acute respiratory syndrome coronavirus vaccine provides incomplete protection in mice and induces increased eosinophilic proinflammatory pulmonary response upon challenge. Journal of Virology, 85(23), 12201-12215.

24. Dan-Dan, L., \& Qi-Han, L. (2021). SARS-CoV-2: Vaccines in the pandemic era. Military Medical Research, 8, 1 . 
25. Zhong, N., Zheng, B. J., Li, Y. M., Poon, L. L. M., Xie, Z. H., Chan, K. H., Li, P. H., Tan, S. Y., Chang, Q., Xie, J. P., Liu, X. Q., Xu, J., Li, D. X., Yuen, K. Y., Peiris, J. S. M., \& Guan, Y. (2003). Epidemiology and cause of severe acute respiratory syndrome (SARS) in Guangdong, People's Republic of China. The Lancet, 362(9393), 1353-1358.

26. Peiris, J. S. M., et al. (2004). Severe acute respiratory syndrome. Nature Medicine, 10(12), 88-97.

27. Pyrc, K., Berkhout, B., \& van der Hoek, L. (2007). Identification of new human coronaviruses. Expert Review of Anti-Infective Therapy, 5(2), 245-253.

28. Prabakaran, P., Xiao, X., \& Dimitrov, D. S. (2004). A model of the ACE2 structure and function as a SARS-CoV receptor. Biochemical and Biophysical Research Communications, 314(1), 235-241.

29. Ghosh, S., et al. (2020). siRNA could be a potential therapy for COVID-19. EXCLI Journal, 19, 528-531.

30. Uludag, H., et al. (2020). Prospects of RNAi therapy of COVID 19. Frontiers in Bioengineering and Biotechnology, 8, 916.

31. Liu, C., Zhou, Q., Li, Y., Garner, L. V., Watkins, S. P., Carter, L. J., Smoot, J., Gregg, A. C., Daniels, A. D., Jervey, S., \& Albaiu, D. (2020). Research and development on therapeutic agents and vaccines for COVID19 and related human coronavirus disease. ACS Central Science, 6(3), 315-331.

32. Wrapp, D., Wang, N., Corbett, K. S., Goldsmith, J. A., Hsieh, C. L., Abiona, O., Graham, B. S., \& McLellan, J. S. (2020). Cryo-EM structure of the 2019-nCoV spike in the prefusion conformation. Science, 367(6483), 1260-1263.

33. Alam, T. (2017). Nanocarriers as treatment modalities for hypertension. Drug Delivery, 24(1), 358-369.

34. Hodgson, J. (2020). The pandemic pipeline. Nature Biotechnology, 38(5), 523-532. https://doi.org/10.1038/ d41587-02000005-z.

35. Conti, D. S., Brewer, D., Grashik, J., Avasarala, S., \& da Rocha, S. R. P. (2014). Poly (amidoamine) dendrimer nanocarriers and their aerosol formulations for siRNA delivery to the lung epithelium. Molecular Pharmaceutics, 11(6), 1808-1822.

36. Pichichero, M. E. (2020). Understanding messenger RNA and other SARS-CoV-2 vaccines. Medscape. https://www.medscape.com/viewarticle/942654\#vp_3.

37. Black, S. (2015). The costs and effectiveness of large phase III pre-licensure vaccine clinical trials. Expert Review of Vaccines, 14(12), 1543-1548.

38. Wouters-wesseling, W., et al. (2002). Effect of a complete nutritional supplement on antibody response to influenza vaccine in elderly people. The Journals of Gerontology. Series A, Biological Sciences and Medical Sciences, 57, 563-566.

39. Imai, Y., Kuba, K., Rao, S., Huan, Y., Guo, F., Guan, B., Yang, P., Sarao, R., Wada, T., Leong-Poi, H., Crackower, M. A., Fukamizu, A., Hui, C. C., Hein, L., Uhlig, S., Slutsky, A. S., Jiang, C., \& Penninger, J. M. (2005). Angiotensin-converting enzyme 2 protects from severe acute lung failure. Nature., 436(7047), $112-116$.

40. Ventura, M. T., Casciaro, M., Gangemi, S., \& Buquicchio, R. (2017). Immunosenescence in aging: between immune cells depletion and cytokines up-regulation. Clinical and Molecular Allergy, 15(1), 21.

41. Savy, M., et al. (2009). Landscape analysis of interactions between nutrition and vaccine responses in children. The Journal of Nutrition, 139, 2154-2218.

42. Arvas, A. (2014). Vaccination in patients with immunosuppression. Turkish Pediatrics, 49(3), 181-185.

43. Keusch, G. T. (2012). Nutritional effects on response of children in developing countries to respiratory tract pathogens: implications for vaccine development. Reviews of Infectious Diseases, 13, 486-491.

44. Omran, G. A. (2019). Hematological and immunological impairment following in-utero and postnatal exposure to aluminum sulfate in female offspring of albino rats. Immunopharmacology \& Immunotoxicology, 41(1), 40-47.

45. Franceschi, C., Salvioli, S., Garagnani, P., de Eguileor, M., Monti, D., \& Capri, M. (2017). Immunobiography and the heterogeneity of immune responses in the elderly: A focus on inflammaging and trained immunity. Frontiers in Immunology, 8, 982.

46. Yan, B. (2019). microRNAs in cardiovascular disease: Small molecules but big roles. Current Topics in Medicinal Chemistry, 19(21), 1918-1947.

47. Liu, J., \& Guo, B. (2004). RNA-based therapeutics for colorectal cancer: Updates and future directions. Pharmacological Research, 152, 104550.

48. Buchholz, U. J., Bukreyev, A., Yang, L., Lamirande, E. W., Murphy, B. R., Subbarao, K., \& Collins, P. L. (2004). Contributions of the structural proteins of severe acute respiratory syndrome coronavirus to protective immunity. Proceedings of the National Academy of Sciences of the United States of America, 101(26), 9804-9809.

49. Tipnis, S. R., Hooper, N. M., Hyde, R., Karran, E., Christie, G., \& Turner, A. J. (2000). A human homolog of angiotensin-converting enzyme: Cloning and functional expression as a captopril-insensitive carboxypeptidase. The Journal of Biological Chemistry, 275(43), 33238-33243. 
50. DeStefano, F., et al. (2019). Principal controversies in vaccine safety in the United States. Clinical Infectious Diseases, 69, 26-731.

51. Skalny, A. V., et al. (2020). Zinc and respiratory tract infections: Perspectives for COVID-19 (review). International Journal of Molecular Medicine, 46, 17-26.

52. Eliakim, A., Swindt, C., Zaldivar, F., Casali, P., \& Cooper, D. M. (2006). Reduced tetanus antibody titers in overweight children. Autoimmunity., 39(2), 137-141.

53. Calina, D., et al. (2016). Etiological diagnosis and pharmaco therapeutic management of parap-neumonic pleurisy. Farmacia., 64, 946-952.

54. Lee, N., et al. (2006). Anti-SARS-CoV IgG response in relation to disease severity of severe acute respiratory syndrome. Journal of Clinical Virology, 35(2), 179-184.

Publisher's Note Springer Nature remains neutral with regard to jurisdictional claims in published maps and institutional affiliations. 